\title{
PERSUASIÓN A TRAVÉS DE FACEBOOK DE LOS CANDIDATOS EN LAS ELECCIONES GENERALES DE 2016 EN ESPANA
}

\author{
Persuasion through Facebook of the four main \\ candidates in the general election of 2016 in Spain
}

\section{Paloma Abejón-Mendoza y Javier Mayoral-Sánchez}

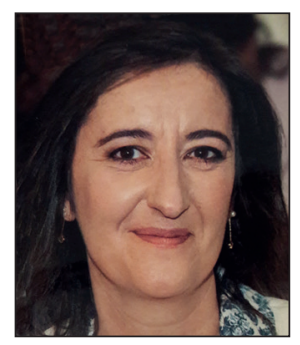

Paloma Abejón-Mendoza es profesora ayudante doctor en el Departamento de Periodismo I de la Universidad Complutense de Madrid (UCM) y doctora en Periodismo. Su trayectoria profesional ha estado vinculada a los gabinetes de comunicación política y al periodismo diario. Desde 2005 imparte clases de redacción periodística en la UCM y es miembro de varios equipos de investigación centrados en redes sociales y la desafección de la política por parte de los ciudadanos. Ha participado en congresos nacionales e internacionales con ponencias relacionadas con sus especialidades y es autora de varios libros.

http://orcid.org/0000-0001-9960-5154

pabejonm@ucm.es

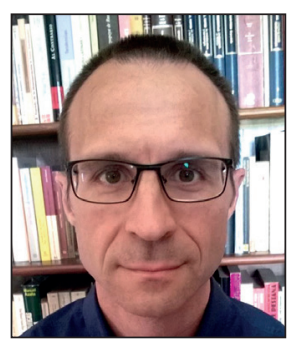

Javier Mayoral-Sánchez es doctor en Periodismo por la Universidad Complutense de Madrid y licenciado en Filología Hispánica. Desde 2000 imparte clases en el Departamento de Periodismo I de la Facultad de Ciencias de la Información de la Universidad Complutense de Madrid (UCM). También fue profesor en la Universidad Camilo José Cela. Es autor entre otros textos de Redacción periodística: medios, géneros y formatos (2013), El uso periodístico de las fuentes en radio y televisión (2009) y Redacción periodística en televisión (2008).

http://orcid.org/0000-0002-7371-1925

javier.mayoral@ucm.es

Universidad Complutense de Madrid, Facultad de Ciencias de la Información Departamento de Periodismo I Avda. Complutense, s/n. 28040 Madrid, España

\section{Resumen}

La persuasión es un proceso comunicativo cuya finalidad es conseguir la adhesión del receptor e influir en él, y este proceso cobra especial relevancia en comunicación política en períodos electorales. El objeto de esta investigación es analizar las técnicas de persuasión utilizadas en Facebook por los candidatos de los principales partidos políticos ( $P P, P S O E, P o d e m o s$ y Ciudadanos) durante la campaña para las elecciones generales de junio de 2016 en España, las últimas celebradas. La investigación revela que, de entre las técnicas persuasivas formuladas por Roiz (1994), las más empleadas por los candidatos a la presidencia del Gobierno en España han sido la repetición de temas, el apoyo en las actitudes preexistentes, la adecuación de los temas a un público concreto y la explotación del contagio psíquico.

\section{Palabras clave}

Persuasión; Elecciones; Campañas electorales; Redes sociales; Comunicación política; Facebook.

\section{Abstract}

Persuasion is a communicative process in which the agent obtains the adhesion of the receiver and influences him/her; this process is particularly relevant in political communication during electoral periods. The persuasive techniques used on Facebook by the candidates of the main political parties ( $P P, P S O E$, Podemos and, Ciudadanos) during the campaign for the general elections of June 2016 in Spain were analyzed. The research reveals that, of all the persuasive techniques envisaged by Roiz (1994), the most employed ones, by candidates to the presidency of the Government in Spain, were topic repetition, support in the preexisting attitudes, the adequacy of the subjects to specific publics, and exploitation of the psychic contagion.

\section{Keywords}

Persuasion; Elections; Electoral campaigns; Social networks; Political communication; Facebook. 
Abejón-Mendoza, Paloma; Mayoral-Sánchez, Javier (2017). “Persuasión a través de Facebook de los candidatos en las elecciones generales de 2016 en España”. El profesional de la información, v. 26, n. 5, pp. 928-936.

https://doi.org/10.3145/epi.2017.sep.14

\section{Introducción}

El barómetro del Centro de Investigaciones Sociológicas (CIS) publicado en julio de 2016 ofrece un dato revelador: en la franja de edad entre 18 y 24 años, en España se informaron tantas personas sobre la campaña electoral del $26 \mathrm{~J}$ a través de redes sociales $(36,6 \%)$ como a través de las webs de medios de comunicación (mismo porcentaje). La cifra desciende según avanzan los tramos de edad hasta el 10,9\% de los mayores de 65 años, pero ofrece una visión sobre el futuro de las campañas electorales en nuestro país.

La mayoría de estudios sobre uso de las redes en campañas electorales tratan de las funciones de éstas, y de los contenidos de los posts (Padró-Solanet, 2010; Carlson, 2008; Williams; Gulati, 2007; 2009; Norris; Curtice, 2006; Anduiza et al., 2010), y no hay investigaciones centradas en analizar los mensajes desde el punto de vista de la redacción periodística y de las técnicas de persuasión utilizadas.

\section{La comunicación persuasiva intenta cap- tar la atención del receptor, muchas ve- ces de forma estridente y provocadora}

La comunicación persuasiva es cada día más frecuente en política y pone todos los medios a su alcance para captar la atención del receptor, muchas veces de forma estridente y provocadora, como confirman Gallardo y Enguix (2016), que señalan dos situaciones perversas: el recurso al "habla peligrosa" (Benesch, 2012) y la irrelevancia de la política (Innerarity, 2004). Lo demuestra la victoria de Donald Trump en las elecciones de Estados Unidos, utilizando un perfil en las redes que encaja perfectamente en ambas perversiones.

La política se ha vuelto más emocional que racional sobre los programas y candidatos, y se debería buscar el punto medio entre ambos aspectos. Dader (1992; 2008) ya adelantó hace unos años que, cuando el intercambio de discursos de la comunicación política se reduce a una batalla entre relatos lanzados con criterios emocionales para seducir, siguiendo estrategias de marketing político, la veracidad de los hechos queda oscurecida. Advertía sobre la llamada "posverdad" antes de su aparición.

Esta investigación se centra en el análisis del uso de Facebook por los candidatos a las elecciones del 26 de junio de 2016. Se opta por esta red social porque, según coinciden en afirmar varios trabajos (IAB Spain, 2017), es la más usada en todas las franjas de edad en España y goza de un alto nivel de penetración en todas las capas sociales e ideológicas. Según la propia empresa, cuenta con unos 21 millones de usuarios en nuestro país. En cambio, Comscore (2016) contabiliza 11 millones usuarios de Twitter en España en 2016.
Pese a ello, los principales líderes políticos españoles infrautilizaron Facebook y se inclinaron más por Twitter (Chaves-Montero, 2016, p. 37; González, 2015) en los procesos electorales celebrados en España en 2014-2015: elecciones andaluzas, catalanas y primeras elecciones generales. En 2016, ante la obligación de repetir los comicios generales debido a que no fue posible formar gobierno, los candidatos variaron sus estrategias y comenzaron a trabajar más las acciones en Facebook.

\section{Comunicación persuasiva en política}

Los expertos sitúan el comienzo de la comunicación persuasiva de campañas electorales en las elecciones presidenciales americanas de 1952, que llevaron a Eisenhower a la Casa Blanca a pesar de ser considerado un mal candidato, gracias a la labor de la agencia de relaciones públicas $B B D O$.

La presente investigación se centra en el concepto de persuasión aplicado a la comunicación política como herramienta para lograr un cambio de conducta que favorezca el objetivo del político-persuasor. Compartimos la creencia de Reardon (1981) de que la persuasión no es, como muchos piensan, una actividad carente de ética, sino que se sitúa como una forma de comunicación en la que participa toda persona que entra en relación con los demás, en un marco social en el que todos diferimos en nuestros objetivos y en los instrumentos utilizados para conseguirlos.

Aunque con frecuencia se han destacado sus usos perversos (Brown, 1968), la persuasión no es mala per se, sino la forma de resolver un conflicto convenciendo a nivel comunicativo a alguien para que redefina su objetivo o lo modifique. Traducido al lenguaje electoral, es la forma de convencer a un indeciso. En sentido estricto, toda comunicación política es comunicación estratégica:

"Quien comunica la política lo hace para lograr algo y siguiendo un plan. De manera que la comunicación política es persuasiva e intencionada: está diseñada para influir en una creencia, una actitud, valor o acción" (Canel, 1999, p. 24).

La orientación persuasiva de la comunicación política forma parte por tanto de la esencia misma de la democracia (Martín-Salgado, 2002). Especialmente en el contexto de campañas electorales, convertidas como señala Del-Rey-Morató (2011, p. 113) en

"un relato en el que compiten varios relatos".

Incluso en ese marco las nuevas tecnologías permiten entender la persuasión al modo clásico: no como la imposición de contenidos y argumentos a una audiencia pasiva, sino como un

"intercambio bilateral, un método de aprendizaje mutuo mediante el discurso" (Majone, 1997). 
Investigaciones como las de Lyengar y Kinder (1987) analizan los factores que influyen en los juicios que el público formula sobre los líderes políticos y concluyen que cuando a un votante se le pide un juicio sobre un político éste recurre a la información que tiene más asequible. De ahí la importancia de la información inmediata que recibe el público a través de las redes durante las campañas electorales.

La explicación del efecto de los mensajes persuasivos se ha estudiado desde varias perspectivas teóricas, como la Teoría de la respuesta cognitiva, el Modelo heurístico o el Modelo de probabilidad de elaboración (Petty; Cacioppo, 1986). Este último modelo permite la integración de los dos anteriores y subraya que cuando recibimos un mensaje persuasivo podemos analizarlo racionalmente y responder automáticamente o generar un cambio de actitud. En las dos opciones, la decisión depende de dos factores: la motivación y la capacidad para hacerlo.

\section{Los principales líderes políticos españo-} les infrautilizaron Facebook y se inclinaron más por Twitter en los procesos electorales celebrados en España en 2014-2015

El fenómeno persuasivo está cada vez más orientado hacia la aplicación de técnicas y procedimientos (argumentación, semantización, aplicación de principios psicológicos) cuyo fin es lograr convencer (Roiz, 2002). Según este autor las principales técnicas de persuasión son seis (Roiz, 1994):

- apelación a los sentimientos;

- simplificación del mensaje;

- desfiguración del sentido original de la información;

- repetición de temas e ideas adecuados a diferentes públicos;

- explotación del contagio psíquico;

- apoyo en las actitudes preexistentes.

Éstas son las técnicas en las que se basa esta investigación.

La mayoría de los políticos españoles centran sus campañas en incrementar al máximo los niveles de compromiso o engagement (Túñez; Sixto, 2011) en las redes sociales, ya que la política no es otra cosa que conectar personas, y en la Red sigue siendo eso, conseguir que esas personas conecten con otras (Peytibi, 2011). La eficacia de los argumentos utilizados se mide más por el grado de aceptación que tengan que por su veracidad, por lo que el factor emocional que facilita esa aceptación cobra relevancia y las técnicas de persuasión pasan a ser primordiales.

Aunque la campaña emocional más referida por todos los estudios es la de Obama de 2008 (Hendricks; Denton 2010; Vaccari, 2010), esta investigación pretende sumarse a estudios que intentan comprender las estrategias de comunicación online partiendo de una discusión más general sobre la modernización de los procesos electorales (Mazzoleni, 2010; Norris, 2011; Dader et al., 2011), y desde la perspectiva de la redacción periodística y de las técnicas de persuasión utilizadas.

\section{Metodología y objetivos}

Realizamos una investigación cuantitativa, en la que se busca delimitar los elementos de persuasión utilizados por cada candidato, para intentar desentrañar estrategias basadas en las técnicas de persuasión más usadas y también medir su efectividad en relación con el compromiso que generan, una cuestión esencial para los políticos en los procesos electorales.

Se decidió elegir a cuatro dirigentes políticos:

-Mariano Rajoy (Partido Popular, PP);

-Pedro Sánchez (Partido Socialista Obrero Español, PSOE);

-Albert Rivera (Ciudadanos, C's);

-Pablo Iglesias (Podemos).

La justificación es que eran los líderes de los partidos que, conforme a los resultados de las elecciones de diciembre de 2015 y según la encuesta del CIS publicada el 9 de junio de 2016, tenían más posibilidades de ganar las elecciones generales del 26 de junio. Se centró el análisis en una semana de precampaña (del 5 al 11 de junio de 2016) y otra semana de campaña, previa al día de los comicios (del 19 al 25 de junio de 2016) con el fin de analizar si había variaciones en las técnicas de persuasión según se iba acercando la fecha de la contienda electoral.

Se buscó en los posts de los candidatos, no de los partidos, las siete técnicas generales de persuasión determinadas por Roiz (1994) señaladas antes. Para la recopilación del material de trabajo se recurrió a Smétrica. Esta empresa cuenta con una aplicación gratuita de mediciones en Facebook que tiene en seguimiento más de 16.000 páginas, de las que recogen diariamente el número de fans, "hablando de esto", publicaciones, comentarios, etc. Sin embargo, para la elaboración de este estudio se contrató un servicio no gratuito que permite búsquedas más específicas y amplias. Desde el punto de vista metodológico, no se trata de una encuesta ni de un sistema de proyección de datos, sino de la extracción selectiva de contenidos publicados en Facebook. Cada uno de los comentarios analizados puede recuperarse si se desea, accediendo a las webs citadas.

Smétrica realiza el proceso de extracción de datos con un script programado en Python, y los datos provenientes de Facebook fueron consultados a través de su API.

https://www.python.org

https://developers.facebook.com/docs/graph-api

Para generar los ficheros de salida o resultados se decidió que para cada página objetivo se extrajeran los ids de cada publicación o post en el rango de fechas elegido. Para cada uno se hizo una consulta para extraer:

- id de la publicación

- fecha

- texto de la publicación

- id y nombre del autor en Facebook

- tipo de publicación.

Además, para cada publicación se extrajeron los siguientes datos sobre los comentarios:

- id del comentario

- fecha 
Tabla 1. Posts de los candidatos y comentarios recibidos

\begin{tabular}{|l|c|c|}
\cline { 2 - 3 } \multicolumn{1}{c|}{} & $\begin{array}{c}\text { Número de } \\
\text { posts }\end{array}$ & $\begin{array}{c}\text { Comentarios } \\
\text { recibidos }\end{array}$ \\
\hline Mariano Rajoy (PP) & 29 & 6.142 \\
\hline Pedro Sánchez (PSOE) & 40 & 8.751 \\
\hline Pablo Iglesias (Podemos) & 31 & 50.641 \\
\hline Albert Rivera (Ciudadanos) & 13 & 2.962 \\
\hline Total & 113 & 68.496 \\
\hline
\end{tabular}

- texto del comentario

- id y nombre del autor

- número de réplicas realizadas por la página

- número de réplicas realizadas por usuarios

- comentarios totales (página y usuarios).

\section{Desarrollo de la investigación}

El corpus de esta investigación lo constituyen los 113 posts enviados a través de su Facebook por los cuatro candidatos elegidos en las dos semanas establecidas, del 5 al 11 de junio de 2016 y del 19 al 25 de junio de 2016, así como 4.125 de los comentarios que generaron. Analizar la totalidad de los 68.496 comentarios recibidos por los líderes (tabla 1) era un objetivo inalcanzable, así que se determinó limitar el análisis a los posts que superaran los 100 comentarios y de éstos únicamente los 25 primeros de los que recibían entre 100 y 200 comentarios y los 50 primeros de los que superaran los 200. En total se han revisado 4.125 comentarios (tabla 2):

El análisis cuantitativo se refiere únicamente a los 113 posts, mientras que los comentarios han sido analizados desde el
Tabla 2. Número de comentarios analizados por cada candidato

\begin{tabular}{|l|c|c|r|}
\hline \multicolumn{1}{|c|}{ Candidato } & 1a semana & 2a semana & Total \\
\hline Mariano Rajoy & 350 & 575 & 925 \\
\hline Pedro Sánchez & 475 & 750 & 1.225 \\
\hline Pablo Iglesias & 850 & 650 & 1.500 \\
\hline Albert Rivera & 225 & 250 & 475 \\
\hline Total & 1.900 & 2.225 & 4.125 \\
\hline
\end{tabular}

punto de vista cualitativo con el objetivo de extraer líneas de actuación llevadas a cabo tendentes a potenciar las estrategias de sus líderes.

Conviene precisar que para medir el efecto de los posts nos centramos en uno de los tres componentes clásicos del engagement (compromiso): los comentarios, prescindiendo de likes y compartidos. La decisión se tomó en base a la utilidad para la investigación. Para analizar las técnicas de persuasión debíamos fijarnos no sólo en los posts de los candidatos, sino también en las estrategias de sus equipos y la única forma de hacerlo era a través de los comentarios, ya que en likes o compartidos no es posible analizar técnica alguna, al ser reacciones inmediatas en las que no se puede medir ni apreciar elementos persuasivos.

El PP fue el partido con una apuesta más clara por Facebook en la campaña electoral del 26J. Contrató los servicios de la empresa The Messina Group (TMG), que había sido la encargada de la campaña de reelección de Obama en 2012, y que planteó una estrategia basada en el análisis de datos masivos (big data, o sea gestionar y procesar la información a partir de grandes cantidades de datos) para captar electores.

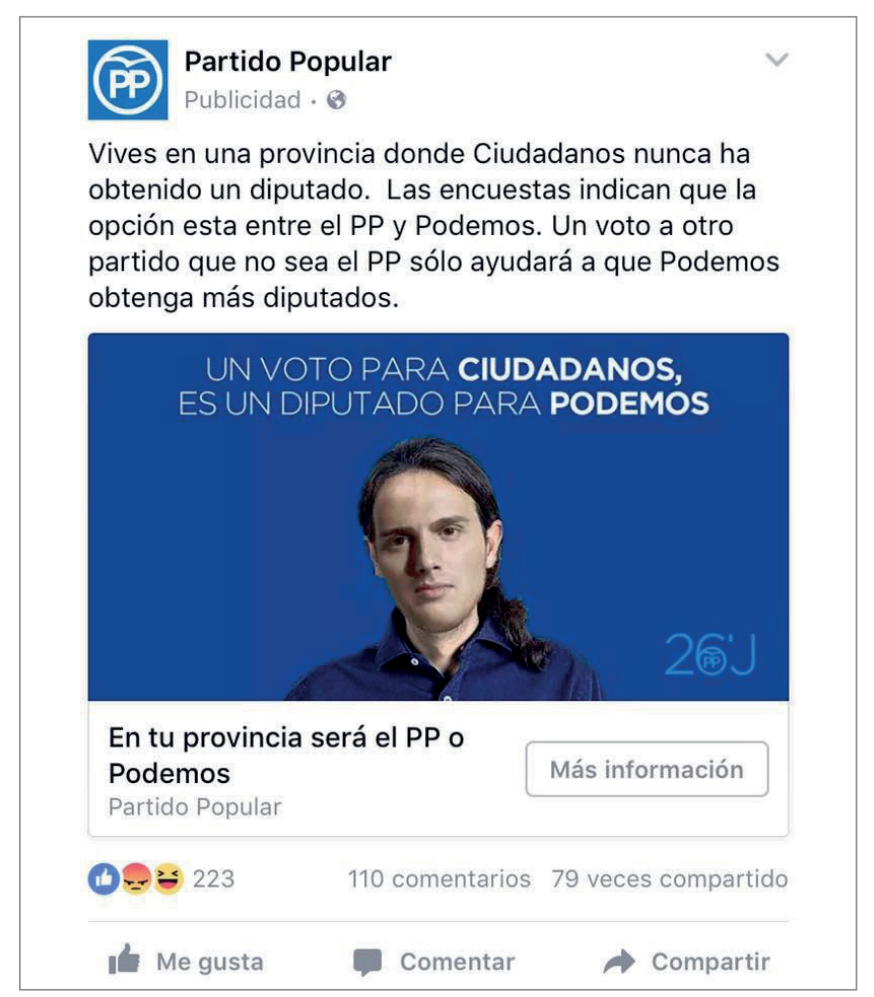

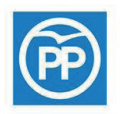

\section{Partido Popular}

Publicidad $\cdot 6$

Vives en una provincia donde Ciudadanos nunca ha obtenido un diputado. Las encuestas indican que la opción esta entre el PP y Podemos. Un voto a otro partido que no sea el PP sólo ayudará a que Podemos obtenga más diputados.

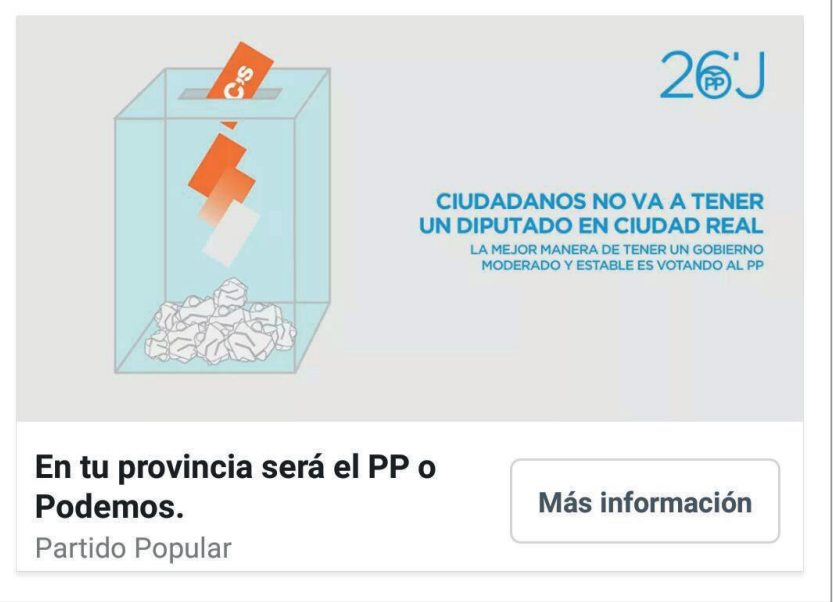

Figura 1. Posts del PP en Facebook dirigido a los indecisos en las provincias en las que, si se dividía el voto del centro derecha, el escaño se lo podía llevar Podemos 
Definieron públicos muy claros localizándolos por sus perfiles de Facebook y buscaron un objetivo: los votantes de Ciudadanos principalmente. La intención de su campaña en Facebook era captar el voto de 2 millones de indecisos en las provincias en las que, si se dividía el voto del centro derecha, el escaño se lo podía llevar Podemos. Un ejemplo de este tipo de entradas que hizo el $P P$ en las redes es una fotografía en la que aparece la cara de Albert Rivera con la coleta de Pablo Iglesias con la leyenda: "Un voto para Ciudadanos, es un diputado para Podemos" y "En tu provincia será el $P P$ o Podemos" (figura 1).

Los posts de Mariano Rajoy en las semanas analizadas van en la línea de repetir hasta la saciedad esta idea (técnica de persuasión de Roiz). Rajoy insistía una y otra vez: "si votas a Ciudadanos, no sabes quién va a acabar gobernando; si votas al PP, sabes lo que votas". Así, lo que Albert Rivera vendía como una virtud, la capacidad de pactar, era convertido por el $P P$ en una debilidad.

\section{La eficacia de los argumentos utilizados se mide más por el grado de aceptación que por su veracidad}

Rajoy prácticamente obvió a Pedro Sánchez y combinó la repetición de ideas con la adecuación de temas a su espectro de votantes, pero también a aquellos que temían la irrupción de Podemos por ser personas moderadas. Por ejemplo, Rajoy hizo diversas referencias a Venezuela: "España no es Venezuela", "Los radicalismos nos llevan a la catástrofe".

El $50 \%$ de sus posts introducía vídeos, más en la semana analizada de campaña electoral que en la de precampaña. La agencia de publicidad encargada de la campaña entendió que Rajoy tenía que romper con la imagen clásica de su partido y apostó por vídeos en los que la técnica más utilizada era la apelación a los sentimientos, algo que el candidato prácticamente no utiliza en sus posts.

Los vídeos de Rajoy también utilizaron otra técnica de persuasión: la de adecuar los temas a un tipo concreto de público. Conscientes del perfil de mayor edad de sus votantes, lanzaron la campaña "Piensa sin prejuicios", que ya habían iniciado

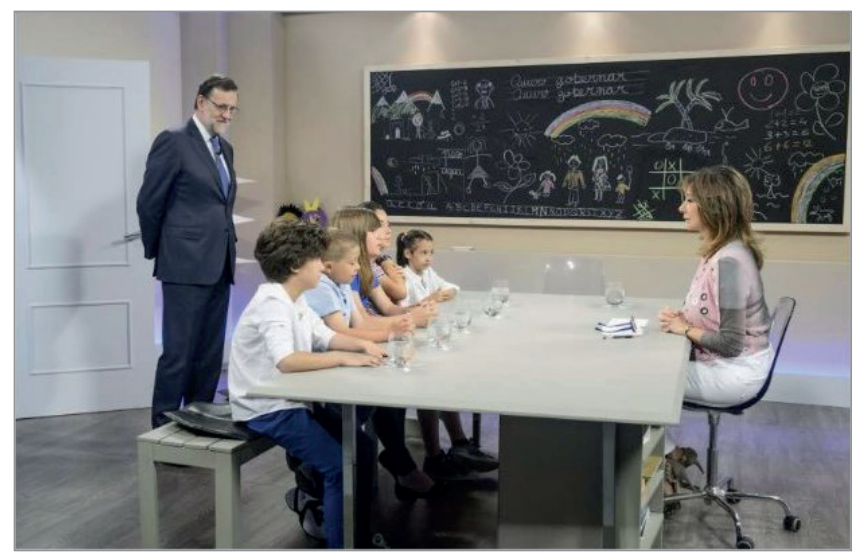

Figura 2. Mariano Rajoy en el programa de Telecinco "Quiero gobernar" http://www.20minutos.es/noticia/2767931/0
Tabla 3. Técnicas utilizadas por Mariano Rajoy y número de comentarios

\begin{tabular}{|l|c|c|}
\cline { 2 - 3 } \multicolumn{1}{c|}{} & $\begin{array}{c}\text { Posts en los } \\
\text { que se utiliza }\end{array}$ & $\begin{array}{c}\text { Media de } \\
\text { comentarios } \\
\text { recibidos }\end{array}$ \\
\hline Apelación a los sentimientos & 10 & 281 \\
\hline Simplificación del mensaje & 5 & 203 \\
\hline Desfiguración del sentido original & 1 & 134 \\
\hline Repetición de temas & 15 & 259 \\
\hline $\begin{array}{l}\text { Adecuación de temas a un público } \\
\text { concreto }\end{array}$ & 11 & 211 \\
\hline Contagio psíquico & 8 & 168 \\
\hline Apoyo en actitudes preexistentes & 10 & 247 \\
\hline
\end{tabular}

en las elecciones del 20 de diciembre con el vídeo del Hipster. https://youtu.be/W_VQbsuRXI4

En la campaña del 26J optaron por el vídeo Abuelas, en el que una joven explicaba cómo consideraba a su abuela un ejemplo a seguir y lanzaba la pregunta: ¿Por qué rechazas de entrada al partido al que vota?

https://youtu.be/AUljMgOyjuO

Para entender bien el éxito o no de las técnicas de persuasión utilizadas, hay que precisar que la media de comentarios que recibió Rajoy a sus posts la primera semana del análisis fue de 210 y la segunda semana de 212, así como que el número de técnicas de persuasión que aparecen en las tablas personalizadas de los candidatos supera al número total de posts que aparece en la tabla 1 porque en algunos posts usan más de una técnica (tabla 3 ).

Las entradas de Rajoy que mayor número de comentarios, likes y compartidos generaron coinciden con aquellas en las que el líder popular usó la apelación a los sentimientos, una técnica de persuasión que utilizó muy pocas veces y no siempre con soltura, pero que sin embargo le dio estupendos resultados. En la primera semana de análisis (5-11 junio 2016), el post que más repercusión tuvo en las redes fue uno del 8 de junio en el que el presidente en funciones hablaba de lo bien que se lo había pasado en el programa de Telecinco "26J. Quiero gobernar" en el que varios niños le hacían preguntas. Pulverizó todos sus récords de posts en la

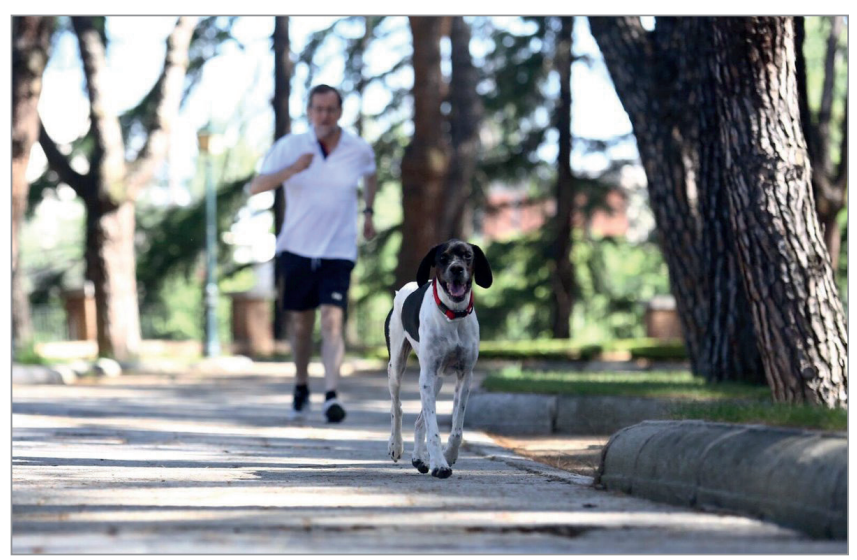

Figura 3. https://pbs.twimg.com/media/ClyoYNdWYAAAqMn.jpg 
Red en los dos períodos estudiados, 407 comentarios, 5.316 likes y 2.826 compartidos.

En su segunda semana, Rajoy logró su récord con un post el día de la jornada de reflexión en el que hacía un comentario totalmente personal: "Un poco de deporte con mi perro Rico antes de pasar un agradable día en familia". Logró 830 comentarios, 447 compartidos y más de 7.000 likes (figura 3).

El líder del PSOE, Pedro Sánchez, fue el que más contenido generó en Facebook. Sus posts son excesivamente largos, y la técnica de la simplificación del mensaje no la trabaja, apenas la hemos encontrado en 2 de los 40 mensajes que emitió durante las dos semanas analizadas. La totalidad del contenido de sus posts supera en 4,5 veces la extensión de los mensajes del resto de los candidatos.

Sánchez ha manejado esencialmente tres técnicas de persuasión: repetición de temas, contagio psíquico y actuar sobre las actitudes preexistentes.

Son muy numerosas sus apelaciones a los "socialistas de corazón", "a los que queréis que no gobierne la derecha en España". Su posible electorado estaba siendo tentado por otras opciones y debía apelar a la tradición socialista como arma para intentar frenar la fuga de votos que le auguraban las encuestas. Es precisamente esta última técnica la que mejor resultado le dio a juzgar por los comentarios recibidos en este tipo de post: 297 comentarios de media, muy por encima de los 206 comentarios de media en la primera semana y de los 226 de la segunda semana de su standard que veremos más adelante.

El líder socialista, igual que Rajoy, apenas apela a los sentimientos en sus posts, y cuando lo hace es más bien priorizando la técnica del contagio psíquico, una de las que más ha utilizado:

"Os pido que el próximo domingo salgáis a votar al PSOE con orgullo, con razón e ilusión. Hagamos de nuestro voto una reivindicación de nuestro pasado, de nuestro presente y de nuestro futuro. iGanemos las elecciones! ¡A ganar! ¡A ganar! ii A ganar!!!”.

Pablo Iglesias se ha mostrado como un candidato que en Facebook trata de buscar mensajes emotivos con los que el votante se identifique, ilusione, entusiasme y movilice. Por eso su engagement es el mayor de todos, como demuestra la tabla 1. La media de comentarios que ha recibido el líder de la formación morada en la primera semana del estudio es de 1.698 y la segunda fue de 1.544 .

Se puede decir que Iglesias utilizó todas las técnicas de persuasión de Roiz en la campaña del 26 de junio. Entre ellas apostó por una que prácticamente no utilizó ninguno de los otros líderes, la simplificación del mensaje. Sus posts son cortísimos y su efectividad es máxima:

“En el Día Mundial de los Refugiad@s... Quo vadis, Europa?".

Su reiteración de temas se centraba en acabar con los recortes y en mostrar a Podemos como un par-
Tabla 4. Técnicas utilizadas por Pedro Sánchez y número de comentarios

\begin{tabular}{|l|c|c|}
\cline { 2 - 3 } \multicolumn{1}{c|}{} & $\begin{array}{c}\text { Posts en los } \\
\text { que la utiliza }\end{array}$ & $\begin{array}{c}\text { Media de } \\
\text { comentarios } \\
\text { recibidos }\end{array}$ \\
\hline Apelación a los sentimientos & 9 & 241 \\
\hline Simplificación del mensaje & 0 & - \\
\hline Desfiguración del sentido original & 1 & 134 \\
\hline Repetición de temas & 25 & 249 \\
\hline $\begin{array}{l}\text { Adecuación de temas a un públi- } \\
\text { co concreto }\end{array}$ & 11 & 211 \\
\hline Contagio psíquico & 17 & 228 \\
\hline Apoyo en actitudes preexistentes & 19 & 297 \\
\hline
\end{tabular}

tido distinto a los tradicionales y, por ende, limpio de corrupción. Utilizó sobre todo mucha apelación a los sentimientos:

"Esta semana el escenario es todo vuestro. Vosotr@s saltaréis a las tablas para la obra de vuestra vida"

"Hay abrazos que, sin hablar, lo dicen todo".

Los posts de Iglesias trabajaron también la adecuación de temas al público:

“¿Rajoy podemita? Vale, pero aquí somos más de La Gozadera"

-referido a este vídeo:

https://www.facebook.com/IglesiasTurrionPablo/ videos $/ 1107176036021468$

“Esto es Podemos. Por un país que esté a la altura de Teresa. \#LaSonrisaDeLaAbuela"

-referido a este vídeo:

https://www.youtube.com/watch?v=W3yJffz97bQ

E incitaron a las masas a través de la técnica del contagio psíquico:

“Owen Jones. Como dices, junt@s construiremos esa Europa para la mayoría y no para las élites" (figura 4)

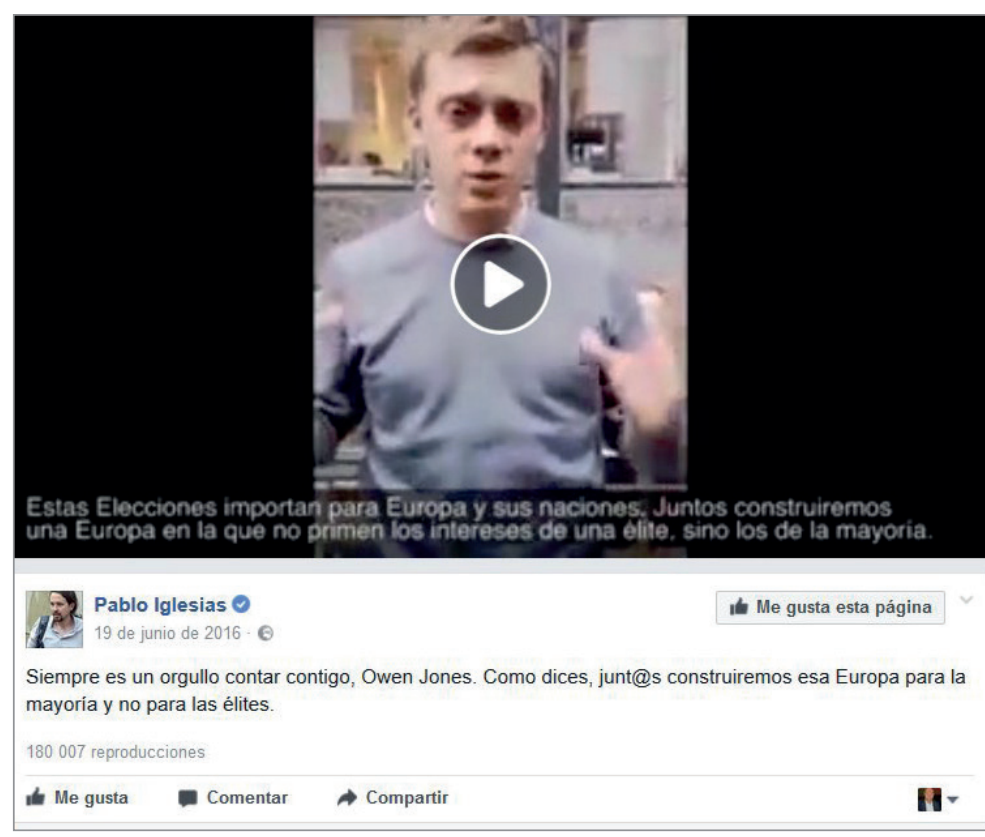

Figura 4. https://www.facebook.com/IglesiasTurrionPablo/videos/1116397768432628 
Tabla 5. Técnicas utilizadas por Pablo Iglesias y número de comentarios

\begin{tabular}{|l|c|c|}
\cline { 2 - 3 } \multicolumn{1}{c|}{} & $\begin{array}{c}\text { Posts en los } \\
\text { que la utiliza }\end{array}$ & $\begin{array}{c}\text { Media de } \\
\text { comentarios } \\
\text { recibidos }\end{array}$ \\
\hline Apelación a los sentimientos & 21 & 2.172 \\
\hline Simplificación del mensaje & 31 & 1.954 \\
\hline Desfiguración del sentido original & 3 & 876 \\
\hline Repetición de temas & 21 & 1.621 \\
\hline $\begin{array}{l}\text { Adecuación de temas a un público } \\
\text { concreto }\end{array}$ & 25 & 1.588 \\
\hline Contagio psíquico & 19 & 1.005 \\
\hline Apoyo en actitudes preexistentes & 20 & 1.849 \\
\hline
\end{tabular}

El último de los candidatos por analizar, Albert Rivera, fue el menos activo en Facebook, sólo 13 posts en las dos semanas analizadas. Además de ser el menos activo también es el que menos técnicas de persuasión utilizó. Sus posts son esencialmente directos y prácticos, pero carentes de la mayoría de las técnicas explicadas por Roiz.

\section{Las publicaciones de los seguidores en Facebook están mucho más cargadas de técnicas de persuasión que los de los lí- deres políticos}

El $77 \%$ de sus posts incluye fotografías, la mayoría en actos y visitas acompañados de agradecimientos (apelación a los sentimientos) y con lemas de campaña, y sólo un $8 \%$ incluye vídeos (spot de campaña \#JuntosSomosImparables).

La repetición de temas ha sido su técnica estrella, muy por encima del resto de las propuestas persuasivas, y si bien sus mensajes utilizan la simplificación del mensaje, pues son todos ellos muy breves, no logran los efectos de engagement que logra con esta técnica Pablo Iglesias porque la mayoría de ellos carecen de cualquier tipo de apelación a las emociones. Sólo en dos posts utiliza esta técnica. En el primero, durante la semana de precampaña, se refiere a su compañera Inés Arrimadas, de la que dice sentirse muy orgulloso y a la que elogia. Es el que más comentarios recibió (659), cuando su media esa semana era de 246 . En el segundo post también se aprecia la técnica del contagio psíquico:

"Porque los españoles, cuando estamos juntos, somos capaces de hacer cosas extraordinarias. Videoclip \#JuntosSomosImparables" (figura 5).

En éste recibe 209 comentarios, algo por encima de su media de esa semana (206 comentarios).

La segunda semana de análisis, los posts de Rivera trabajan, sobre todo, el apoyo a las actitudes preexistentes:

"Orgullosos de ser nuevos, de centro, de querer cambio, de darnos la mano, de ser inconformistas, de ser ciudadanos \#MadridNaranja".
Tabla 6. Técnicas utilizadas por Albert Rivera y número de comentarios

\begin{tabular}{|l|c|c|}
\cline { 2 - 3 } \multicolumn{1}{c|}{} & $\begin{array}{c}\text { Posts en los } \\
\text { que la utiliza }\end{array}$ & $\begin{array}{c}\text { Media de } \\
\text { comentarios } \\
\text { recibidos }\end{array}$ \\
\hline Apelación a los sentimientos & 2 & 434 \\
\hline Simplificación del mensaje & 7 & 226 \\
\hline Desfiguración del sentido original & - & - \\
\hline Repetición de temas & 4 & 325 \\
\hline $\begin{array}{l}\text { Adecuación de temas a un público } \\
\text { concreto }\end{array}$ & - & - \\
\hline Contagio psíquico & 3 & 301 \\
\hline Apoyo en actitudes preexistentes & 3 & 249 \\
\hline
\end{tabular}

\section{Estrategias en los comentarios de posts}

La investigación cualitativa llevada a cabo en el análisis de los 4.125 comentarios que integran el corpus de esta investigación detecta que las publicaciones llevadas a cabo por los seguidores de los líderes políticos están mucho más cargadas de técnicas de persuasión que los posts de los candidatos, que son más asépticos.

La mayoría de los comentarios son claros ejemplos de seguidismo incondicional: "Siempre contigo, Pablo, mi apoyo incondicional", "Rajoy eres el mejor presidente”, etc. Y coinciden con la estrategia de sus líderes de elevar la repetición de temas a la categoría de técnica de persuasión más utilizada. Junto a esa repetición de temas cabe destacar la repetición de palabras exactas en los comentarios, lo que lleva a pensar en estrategias estudiadas.

En los comentarios se detectan muchos elementos del lenguaje emocional, ausentes en los posts originales de los candidatos. Es decir, la apelación a los sentimientos se constituye como la segunda técnica más usada por los seguidores a pesar de que entre los líderes sólo la practica con asiduidad Pablo Iglesias. Los comentarios de los seguidores están llenos de mayúsculas, exclamaciones y emoticonos, aderezados a veces con una llamada a la acción. Esto lleva a que el contagio psíquico se convierta en la tercera técnica

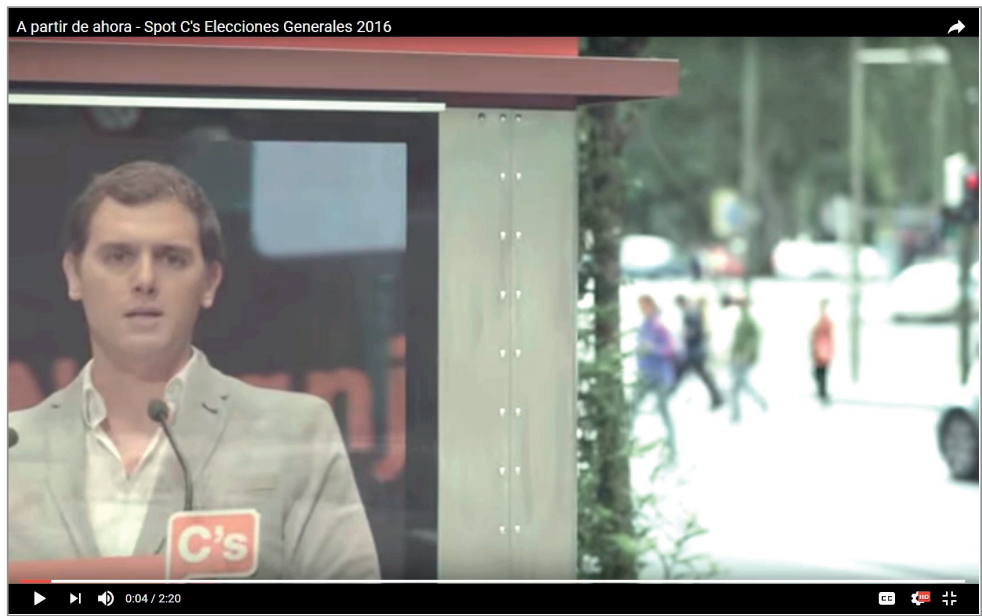

Figura 5. Fotograma del spot de la campaña 2016 \#JuntosSomoslmparables de Ciudadanos.

https://www.youtube.com/watch?v=ovOEnDa-idw 
Tabla 7. Comparativa de las técnicas utilizadas por los candidatos

\begin{tabular}{|c|c|c|c|c|c|}
\hline & Mariano Rajoy & Pedro Sánchez & Pablo Iglesias & Albert Rivera & Total \\
\hline Apelación a los sentimientos & 10 & 9 & 21 & 2 & 42 \\
\hline Simplificación del mensaje & 5 & 0 & 31 & 7 & 43 \\
\hline Desfiguración del sentido original & 1 & 1 & 3 & - & 5 \\
\hline Repetición de temas & 15 & 25 & 21 & 4 & 65 \\
\hline Adecuación de temas a un público concreto & 11 & 11 & 25 & - & 47 \\
\hline Contagio psíquico & 8 & 17 & 19 & 3 & 47 \\
\hline Apoyo en actitudes preexistentes & 10 & 19 & 20 & 3 & 52 \\
\hline Total & 63 & 82 & 142 & 25 & \\
\hline
\end{tabular}

de persuasión más empleada por los seguidores o followers. Los autores de los comentarios arengan a las masas a seguir sus ideas mucho más que los candidatos en sus posts.

\section{Conclusiones}

1) Las técnicas persuasivas de Roiz más empleadas por los candidatos en las elecciones del 26J han sido la repetición de temas, seguida del apoyo en las actitudes preexistentes y, en tercer lugar, empatadas, la adecuación de los temas a un público concreto y el contagio psíquico. Sorprende que la simplificación del mensaje, una técnica de fácil consecución (y una de las de mejores resultados en engagement) sólo ha sido utilizada de forma habitual por Pablo Iglesias.

2) El líder que más técnicas de persuasión utilizó en sus posts fue Pablo Iglesias, seguido de Pedro Sánchez, Mariano Rajoy y, en último lugar, Albert Rivera.

La técnica que más réditos generó a los candidatos en número de comentarios recibidos fue la apelación a los sentimientos

3) La técnica que más réditos generó a los candidatos en cuanto al número de comentarios recibidos fue la apelación a los sentimientos, seguida de la repetición de temas y la simplificación del mensaje. El contagio psíquico, pese a ser una de las más utilizadas, no reportó un incremento significativo en los comentarios recibidos.

4) La combinación de técnicas en un mismo post incrementa de forma significativa sus posibilidades de engagement.

5) El esfuerzo de los líderes políticos en las redes no guarda relación directa con el reconocimiento de los usuarios. Pedro Sánchez, el líder del PSOE, es quien más contenido generó en Facebook, pero el mayor engagement y la mayor audiencia correspondieron a Pablo Iglesias.

6) Las técnicas de persuasión utilizadas no varían entre la precampaña y la campaña.

7) Entre los seguidores de los líderes políticos las técnicas más utilizadas fueron la repetición de temas, seguida de la apelación a los sentimientos y el apoyo en las actitudes preexistentes.

8) En la campaña del 26J los candidatos han buscado las dos principales emociones que se intenta lograr en una campaña electoral: el miedo y el entusiasmo. Después del Brexit, los mensajes del PP se orientaron a crear miedo diciendo que ocurriría lo mismo si ganan las elecciones los "radicales", "extremistas" o "independentistas" de Unidos Podemos, mientras que Podemos y Ciudadanos, las fuerzas emergentes, trabajaron para crear la ilusión del entusiasmo por una nueva etapa.

9) La campaña del 26J en Facebook ha compaginado el factor emocional y la segmentación de los destinatarios de los mensajes, siguiendo la tendencia de campañas americanas.

La persuasión, traducida al lenguaje electoral, es la forma de convencer a un indeciso

\section{Apoyos}

Esta investigación se enmarca dentro del proyecto Las estrategias de campaña online de los partidos políticos españoles 2015-2016 de la Universidad Complutense de Madrid, dirigido por el catedrático de Periodismo José-Luis Dader y financiado por el Ministerio de Economía de España.

Con cargo a este proyecto se contrataron los servicios de la empresa encargada de la recogida de datos del Facebook de los candidatos, Smétrica, que ofrece la posibilidad de solicitar búsquedas específicas más ampliadas que las de la aplicación gratuita.

\section{Bibliografía}

Anduiza, Eva; Cantijoch, Marta; Colombo, Clelia; Gallego, Aina; Salcedo, Jorge (2010). "Los usos políticos de Internet en España". Revista española de investigaciones sociológicas, n. 129, pp. 133-146.

http://www.reis.cis.es/REIS/PDF/REIS_129_051262609304456. $p d f$

Benesch, Susan (2012). Dangerous speech: A proposal to prevent group violence. World Policy Institute. https://goo.gl/aHda2B

Brown, James (1968). Techniques of persuasion: From propaganda to brainwashing. Londres: Penguin Books. ISBN: 0140206043

Canel, María-José (1999). Comunicación política. Una guía para su estudio y práctica. Madrid: Tecnos. ISBN: 97884 30944408 
Carlson, Tom (2008). "Learning the ropes: The adoption of blogs by political candidates in the 2007 Finnish national elections". En Politics: Web 2.0: An international conference, Londres. http://bit.ly/2qq2FoY

Chaves-Montero, Alfonso (2016). La comunicación política en las redes sociales durante la campaña electoral de 2015 en España. Uso, efectividad y alcance. Universidad Internacional de Andalucía. ISBN: 9788479935931 https://goo.gl/y2nn9X

Comscore (2016). Futuro digital global 2016. Comscore report. https://www.comscore.com/lat/Insights/Presentations-andWhitepapers/2016/2016-Global-Digital-Future-in-Focus

Dader, José-Luis (1992). El periodista en el espacio público. Barcelona: Bosch. ISBN: 9788476762172

Dader, José-Luis (2008). "La adolescente investigación en comunicación política: estructura del campo y tendencias prometedoras". En: Martínez-Nicolás, Manuel (ed.). Para investigar la comunicación. Propuestas teórico-metodológicas. Madrid: Tecnos, pp. 133-177. ISBN: 9788430948215 https://goo.gl/GLEuD8

Dader, José-Luis; Campos-Domínguez, Eva; Vizcaíno-Laorga, Ricardo; Cheng, Lifen (2011). "Las webs de los partidos españoles durante la campaña para las Elecciones Generales de 2008: pautas de cibermarketing con pocos signos de ciberdemocracia". En: Sampedro-Blanco, Víctor (ed.). Cibercampaña, cauces y diques para la participación. Las elecciones generales de 2008 y su proyección tecnopolítica. Madrid: Editorial Complutense. ISBN: 9788499380940

Del-Rey-Morató, Javier (2011). "La comunicación política en la sociedad del marketing y de internet. Encuadres, relatos y juegos de lenguaje". Revista de comunicación, n. 10, pp. 102-128.

https://goo.gl/nK5DWu

Gallardo, Beatriz; Enguix, Salvador (2015). Pseudopolítica: el discurso político en las redes sociales. Valencia: Universitat de València. ISBN: 9788460867371

González, Christian (2015). "Twitter y Facebook, el otro campo de batalla por el 20-D”. Público, 11 diciembre.

https://goo.gl/oY8g7s

Hendricks, John-Allen; Denton, Robert (2010). Communicator-in-chief: How Barack Obama used new media technology to win the White House. Lanham, MD: Lexington Books. ISBN: 9780739141069

IAB (2017). Estudio anual de redes sociales 2017. Madrid: Interactive Advertising Bureau.

https://goo.gl/RT9ASD

Innerarity, Daniel (2004). La sociedad invisible. Madrid: Espasa. ISBN: 9788467015973

Lyengar, Shanto; Kinder, Donald (1987). "News that matters". In: Theodoulou, Stella; Cahn, Matthew. Public policy: The essential readings. Nueva Jersey: Pearson, pp. 295-305. ISBN: 9780205856336

Majone, Giandomenico (1997). Evidencia, argumentación y persuasión en la formulación de políticas. México: Fondo de
Cultura Económica. ISBN: 9681649257

Martín-Salgado, Lourdes (2002). Marketing politico: arte y ciencia de la persuasión en democracia. Barcelona: Paidós. ISBN: 9788449312380

Mazzoleni, Gianpietro (2010). La comunicación política. Madrid: Alianza Editorial. ISBN: 9788420669403

Norris, Pippa (2011). Democratic deficit: Critical citizens revisited. New York: Cambridge University Press. ISBN: 0521127440

Norris, Pippa; Curtice, John (2006). "If you build a political web site, will they come? The internet and political activism in Britain". International journal of electronic government research, v. 2, n. 2, pp. 1-21.

https://goo.gl/CtaZH4

Padró-Solanet, Albert (2010). "Internet and votes: The impact of new ICTs on the 2008 Spanish parliamentary elections". In: Internet, politics, policy 2010: An impact assessment conference, Oxford Internet Institute.

http://ipp.oii.ox.ac.uk/sites/ipp/files/documents/IPP2010_ Padro-Solanet_Paper.pdf

Petty, Richard; Cacciopo, John (1986). The elaboration likelihood model of persuasion. New York: Academic Press. ISBN: 9780813330051

https://goo.gl/pL8MaS

Peytibi, Xabier (2011). "Las relaciones políticas online y el ruido en campaña". En: Rovira, Edgar (coord.). Los límites del 2.0, pp. 9-12. https://goo.gl/k1kF8y

Reardon, Katleen (1981). La persuasión en la comunicación. Barcelona: Paidós Comunicación. ISBN: 9788475092263

Roiz, Miguel (1994). Técnicas modernas de persuasión. Madrid: Eudema. ISBN: 9788436809718

Roiz, Miguel (2002). La sociedad persuasora: control cultural y comunicación de masas. Barcelona: Paidós Ibérica. ISBN: 9788449313165

Túñez, Miguel; Sixto, José (2011). "Redes sociales, política y compromiso 2.0: la comunicación de los diputados españoles en Facebook". Revista latina de comunicación social, v. 66, pp. $210-234$.

https://doi.org/10.4185/RLCS-66-2011-930-210-246

Vaccari, Cristian (2010). "'Technology is a commodity': The internet in the 2008 United States presidential election". Journal of information technology \& politics, v. 7, pp. 318-339. https://doi.org/10.1080/19331681003656664

Williams, Christine B.; Gulati, Girish J. (2007). "Social networks in political campaigns: Facebook and the 2006 midterm elections". In: 2007 Annual meeting of the American Political Science Association, Chicago, August 30- September 2. https://goo.gl/yvWSgC

Williams, Christine B.; Gulati, Girish J. (2009). "Facebook grows up: An empirical assessment of its role in the 2008 congressional elections". In: 2009 Annual meeting of the Midwest Political Science Association, Chicago, April 2-5. https://goo.gl/guroH5 